\title{
Media Synchronization Framework for SVC Video Transport over IP Networks
}

\author{
Kwang-deok Seo ${ }^{1}$, Jin-won Lee ${ }^{1}$, Soon-heung Jung ${ }^{2}$, and Jae-gon Kim ${ }^{2}$ \\ ${ }^{1}$ Computer and Telecommunications Engineering Division, Yonsei Univ., Gangwon, Korea \\ Kdseo@yonsei.ac.kr \\ ${ }^{2}$ Broadcasting Media Research Group, ETRI, Daejeon, Korea \\ \{zeroone, jgkim\}@etri.re.kr
}

\begin{abstract}
This paper proposes an efficient media synchronization framework for SVC video transport over IP networks. To support synchronization between SVC video and audio signals transported over IP networks, RTP/RTCP protocol suite is usually employed. To provide a framework for media synchronization, we suggest an efficient RTP packetization mode and propose a computationally simple RTCP packet processing method. With the suggested RTP packetization mode, layer synchronization among scalable layers of the SVC video can be effectively achieved. Also by adopting the computationally simple RTCP packet processing, we do not need to process every RTCP SR packet for inter-media synchronization between video and audio signals.
\end{abstract}

Keywords: media synchronization, SVC, RTP, RTCP.

\section{Introduction}

SVC (Scalable Video Coding) known as scalable extension of H.264/MPEG-4 AVC is currently standardized in the JVT (Joint Video Team) of the ISO/IEC MPEG and the ITU-T Video Coding Experts Group [4]. SVC aims at achieving both high compression performance and adaptation for video delivery over heterogeneous networks. SVC is based on H.264/MPEG-4 AVC and provides three scalability modes including temporal, spatial, and quality scalability. Unlike the conventional scalability modes supported in the MPEG-2, H.263, and MPEG-4, SVC scalability can provide combined scalability mode in which the three scalability modes can be aggregated to a single SVC bitstream. The general concept for combining temporal, spatial, and SNR scalability in an SVC bitstream is illustrated in Fig. 1, which shows an example GOP structure for SVC with two scalable layers. The SVC bitstream contains two spatial layers: QCIF encoded at $15 \mathrm{fps}$ and CIF encoded at $30 \mathrm{fps}$. Each spatial layer is composed of one base quality layer and one FGS layer for SNR scalability. The dotted arrow in Fig. 1 designates inter-layer prediction to remove redundancy between spatial layers. In temporal dimension, each picture belongs to one temporal layer indicated by the number in the middle of each picture.

To transport SVC video encapsulated in NAL (network abstraction layer) units over Internet Protocol (IP) in real-time, RTP (real-time transport protocol) and RTCP (RTP control protocol) are usually employed. RTP carries the payload with some 
additional information like sequence number and RTP timestamp. RTCP serves for controlling quality of the transmitted data. RTP timestamp begins at a random number and its rate of increment is proportional to its sampling rate [5]. Thus, they do not directly give information on absolute time reference. To synchronize audio and video data, we need to utilize RTCP Sender Report (SR) packet to find out the absolute time information corresponding to each RTP timestamp carried by each RTP packet.

In this paper, we suggest an efficient RTP packetization mode for layer synchronization among scalable layers of SVC video and propose an efficient inter-media synchronization between SVC video and audio. In the proposed inter-media synchronization method, we do not need to process every RTCP SR packet for synchronization. Moreover, it does not require any floating-point operations or any divisions at all. The proposed method will be compared with conventional method [1].

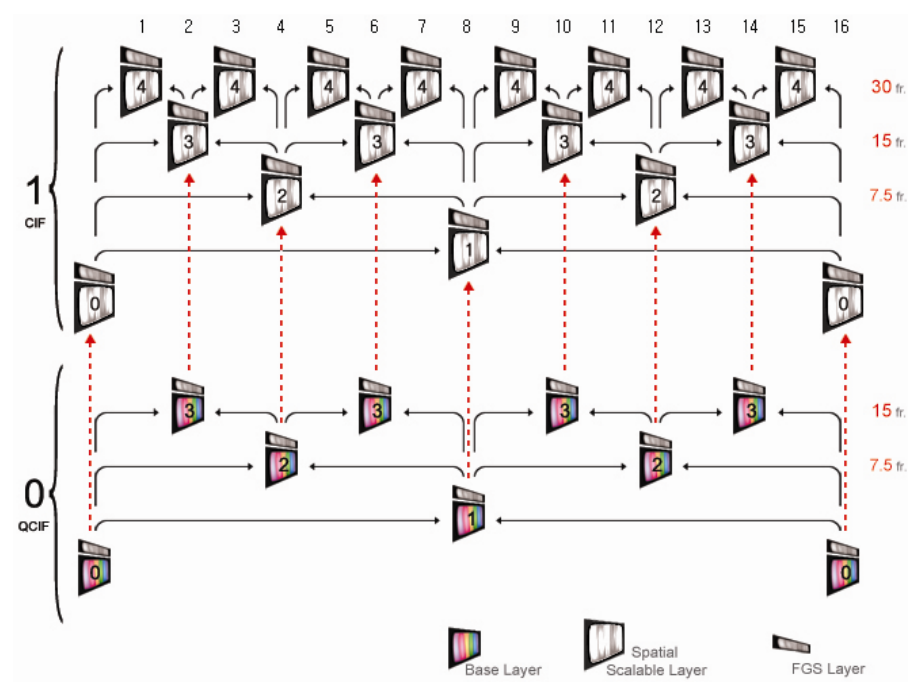

Fig. 1. Combined scalability in an SVC bitstream

\section{Suggested RTP Packetization Mode for Layer Synchronization}

The concepts of VCL (video coding layer) and NAL in SVC are inherited from H.264/MPEG-4 AVC. While the VCL creates a coded representation of the source content, the NAL encapsulates the data generated by the VCL and provides header information in a way that enables simple and effective customization of the use of the VCL for a broad variety of systems. For this purpose, the SVC NAL unit header contains the spatial, temporal, and quality coordinates of the NAL unit payload in the scalability cube which is used for identification and scaling operations of the NAL units. The NAL unit header is designed to co-serve as the payload header of an RTP payload format. For more details on the syntax and semantics of the SVC NAL unit header, you are referred to [3] and [4].

The Audio/Video Transport (AVT) Working Group of the IETF started in November 2005 to draft the RTP payload format for SVC and the signaling for layered 
coding structures [3]. As SVC is a backward compatible extension of H.264, the same should be the case for its RTP packetization. In particular, it is possible to transport the base layer utilizing the same packetization scheme of RFC 3984 [2]. Thus, RFC 3984-aware legacy devices are still capable of utilizing an SVC base layer in an RTP transport environment.

An RTP stream carrying only one layer would carry NAL units belonging to that layer only. An RTP stream carrying a complete scalable video bitstream would carry NAL units of a base layer and one or more enhancement layers. In the former case, however, the system administrator of the server should open a separate UDP port for each RTP session to carry a single layer. Thus, the server should open as many ports as required to transport all the layers. System administrators would like to avoid opening too many UDP ports in their firewalls, because of the security risk and the administrative effort. Moreover, for mass deployment to end terminals, it is desirable to reduce the number of UDP ports in a firewall to the absolute minimum-ideally to a single one. In this respect, the latter approach is much preferred to the former one.

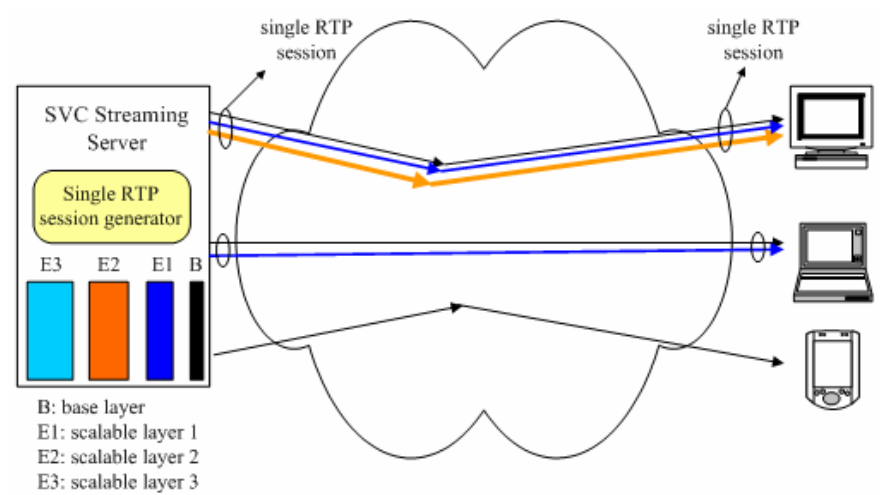

Fig. 2. SVC streaming scenario based on single RTP session

This line of thought leads to the service scenario as depicted in Fig. 2, where the server opens only a single RTP session to carry one or more layers. For each terminal, the server composes a bitstream tailored to the terminal's needs by aggregating NAL units of appropriate layers. 'Single RTP session generator' is used to aggregate the extracted contents from potentially more than one scalable layer into a single RTP stream carrying one or more layers.

In order to support the service scenario shown in Fig. 2, it is necessary to support encapsulating NAL units from multiple SVC layers into a single RTP packet in the payload format. The IETF specification on RTP payload format for SVC contains mechanisms such as STAP (single-time aggregation packet) and MTAP (multi-time aggregation packet) to aggregate more than one NAL unit into a single RTP packet, and another mechanism called FU (fragmentation unit) to split overly large NAL unit into multiple RTP packets [3]. Two fundamentally different packetization modes of operation are supported in [3]: non-interleaved mode and interleaved mode. Table 1 summarizes the allowed packet types for each packetization mode. In non-interleaved 
mode, the NAL units should be aggregated in decoding order by adopting STAP-A, whereas in interleaved mode, NAL units belonging to multiple pictures can be aggregated out of decoding order by adopting STAP-B and MTAP. Non-interleaved mode is intended to avoid excessive RTP/UDP/IP header overhead that would result when encapsulating small NAL units in single NAL unit packets, whereas the interleaved mode provides an error resilience tool against burst errors. STAP-A aggregates NAL units with identical NALU-time, whereas MTAP aggregates NAL units with differing NALU-time. Here, NALU-time is defined as the value that the RTP timestamp would have if that NAL unit would be transported in its own RTP packet. In Fig. 1, pictures belonging to different spatial layer but having the same picture number (or display time) must have the same NALU-time. Thus, by adopting STAP-A, it is far more feasible to provide synchronization between pictures belonging to different spatial layers but with identical NALU-time. Therefore, Non-interleaved mode is more suitable for systems that require very low end-to-end latency and timely synchronization among NAL units from multiple SVC layers aggregated in a RTP packet. Furthermore, as shown in Table 1, only non-interleaved mode supports 'single NAL unit' type that can contain only a single NAL unit in the RTP payload. As a result, noninterleave mode can be suggested as a mandatory packetization mode for fast and real-time streaming requiring timely synchronization among SVC layers, and interleaved mode can be considered as an optional mode for error resilience by providing interleaving function against burst packet loss. In this paper, we employ noninterleaved mode to provide layer synchronization among different scalable layers.

Table 1. Allowed packet types for RTP packetization modes of SVC

\begin{tabular}{clccc}
\hline $\begin{array}{c}\text { NAL Unit } \\
\text { Type }\end{array}$ & $\begin{array}{c}\text { Packet } \\
\text { Type }\end{array}$ & $\begin{array}{c}\text { Single NAL } \\
\text { Unit Mode }\end{array}$ & $\begin{array}{c}\text { Non-interleaved } \\
\text { Mode }\end{array}$ & $\begin{array}{c}\text { Interleaved } \\
\text { Mode }\end{array}$ \\
\hline \hline 0 & undefined & ignore & ignore & ignore \\
$1 \sim 23$ & single NAL unit & yes & yes & no \\
24 & STAP-A & no & yes & no \\
25 & STAP-B & no & no & yes \\
26 & MTAP16 & no & no & yes \\
27 & MTAP24 & no & no & yes \\
28 & FU-A & no & yes & yes \\
29 & FU-B & no & no & yes \\
$30 \sim 31$ & undefined & ignore & ignore & ignore \\
\hline
\end{tabular}

\section{Conventional Inter-media Synchronization Method}

The next problem to resolve for synchronization issue is providing inter-media synchronization between audio and SVC video. According to RFC 3550, it is stated that separate audio and video streams should not be carried in a single RTP session and demultiplexed based on the payload type or SSRC fields [5]. However, we cannot directly use RTP timestamp to synchronize data carried by different RTP sessions for 
the following two reasons. Firstly, RTP timestamp should be initialized to random offsets at session startup to minimize the risk of breaking encryption. Secondly, RTP timestamp increases in proportion to the sampling rate of media. Usually the sampling rates of audio and video data are quite different. Thus, the rates of increase in RTP timestamp for audio and SVC video sessions are not the same. To circumvent these problems, RTCP SR packets carrying both the RTP and the NTP timestamp are generally employed.

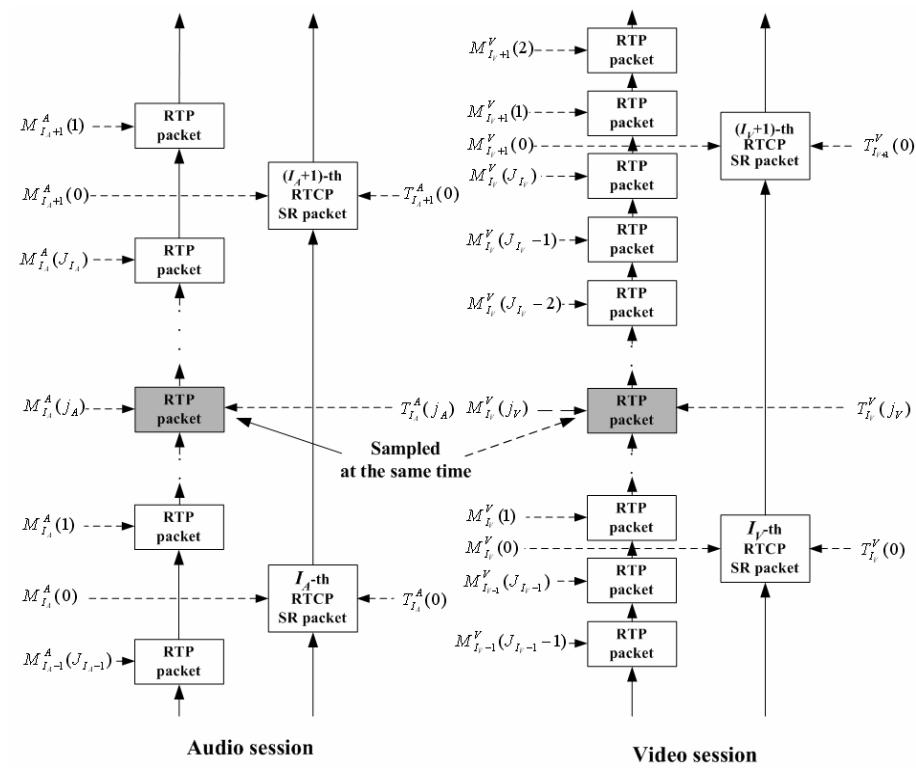

Fig. 3. RTP/RTCP streams for audio and SVC video sessions

Fig. 3 shows RTP and RTCP streams for audio and SVC video sessions. In Fig. 3, each RTP packet of SVC video session aggregates NAL units that all share the same NALU-time. The RTP timestamp of each RTP packet must be set to the NALU-time of all the NAL units to be aggregated. An aggregation packet can carry as many NAL units as necessary. However, the total amount of data in an aggregation packet obviously must fit into an IP packet, and the size should be chosen so that the resulting IP packet is bound by the MTU size of the transport channel.

The superscripts $A$ and $V$ are used to denote audio and SVC video sessions, respectively. For the derivation of the relationship between the RTP timestamp of a specific RTP packet and the absolute time reference, let us consider the shaded RTP packet in the audio session shown in Fig. 3. For this RTP packet, $T_{I_{A}}^{A}\left(j_{A}\right)$ is the absolute time reference for RTP timestamp of the $j_{A}$-th RTP packet after the $I_{A}$-th RTCP packet has been received. NTP (Network Time Protocol) tells us how to set the absolute time information. As a special case, when $j_{A}=0, T_{I_{A}}^{A}(0)$ is the NTP timestamp contained in the $I_{A}$-th RTCP packet. Similarly, $M_{I_{A}}^{A}\left(j_{A}\right)$ is the RTP timestamp contained in the $j_{A}$-th 
RTP packet after the $I_{A}$-th RTCP packet, and $M_{I_{A}}^{A}(0)$ is the RTP timestamp for the $I_{A^{-}}$ th RTCP packet.

Let us assume that the shaded RTP packet in the SVC video session is sampled at the same time with the shaded RTP packet in the audio session. If the absolute time reference of this SVC RTP packet is represented by $T_{I_{V}}^{V}\left(j_{V}\right)$, it is required that $T_{I_{A}}^{A}\left(j_{A}\right)=T_{I_{V}}^{V}\left(j_{V}\right)$ for perfect synchronization. However, the transmission rates of RTP packets are normally not the same for different sessions. Moreover, RTCP packets for each session may be transmitted at different time. Thus, even if $T_{I_{A}}^{A}\left(j_{A}\right)=T_{I_{V}}^{V}\left(j_{V}\right), I_{A}$ and $j_{A}$ of the audio session may not be equal to $I_{V}$ and $j_{V}$ of the SVC video session, respectively. Based on this fact, we can compute $T_{I_{A}}^{A}\left(j_{A}\right)$ of a RTP timestamp by using $M_{I_{A}}^{A}\left(j_{A}\right) \cdot M_{I_{A}}^{A}(0)$ and $T_{I_{A}}^{A}(0)$ values are also used in the computation, which can be obtained by the $I_{A}$-th RTCP packet. In the method proposed in [1], the absolute time reference $T_{I_{A}}^{A}\left(j_{A}\right)$ is obtained by

$$
T_{I_{A}}^{A}\left(j_{A}\right)=T_{I_{A}}^{A}(1)+\sum_{k=2}^{j_{A}} \frac{\Delta M_{I_{A}}^{A}(k)}{R^{A}},
$$

where $R^{A}$ is the sampling rate of audio data. $T_{I_{A}}^{A}(1)$ is obtained by

$$
T_{I_{A}}^{A}(1)=T_{I_{A}}^{A}(0)+\frac{M_{I_{A}}^{A}(1)-M_{I_{A}}^{A}(0)}{R^{A}} .
$$

In (1), $\Delta M_{I_{A}}^{A}(k)$ is the difference between the RTP timestamps of two adjacent RTP packets and is given by

$$
\Delta M_{I_{A}}^{A}(k)=M_{I_{A}}^{A}(k)-M_{I_{A}}^{A}(k-1) .
$$

Computation of (1) and (3) continues until a new RTCP packet is received. After receiving the $\left(I_{A}+1\right)$-th RTCP packet, (1) and (2) are computed again using $T_{I_{A}+1}^{A}(0)$ and $M_{I_{A}+1}^{A}(0)$ carried by this RTCP packet. When computing $T_{I_{A}}^{A}\left(j_{A}\right)$ by (1) in Bertoglio's method, they do not compute the term $\sum_{k=2}^{j_{A}} \frac{\Delta M_{I_{A}}^{A}(k)}{R^{A}}$ directly. Instead, since they already know the value of $T_{I_{A}}^{A}\left(j_{A}-1\right)$, they compute the value by

$$
T_{I_{A}}^{A}\left(j_{A}\right)=T_{I_{A}}^{A}\left(j_{A}-1\right)+\frac{\Delta M_{I_{A}}^{A}\left(j_{A}\right)}{R^{A}} .
$$

The same procedure (1)-(4) can be applied to the SVC video session to obtain $T_{I_{V}}^{V}\left(j_{V}\right)$.

When processing $j_{A}-$ th RTP packet for the audio session and $j_{V}$-th RTP packet for the SVC video session, Bertoglio's decision rule based on $T_{I_{A}}^{A}\left(j_{A}\right)$ and $T_{I_{V}}^{V}\left(j_{V}\right)$ for synchronization is as follows:

$$
\begin{array}{lc}
T_{I_{V}}^{V}\left(j_{V}\right)-T_{I_{A}}^{A}\left(j_{A}\right)>\eta_{+} & : \text {SVC video is ahead of audio, } \\
\eta_{+} \geq T_{I_{V}}^{V}\left(j_{V}\right)-T_{I_{A}}^{A}\left(j_{A}\right) \geq-\eta_{-} & : \text {audio and SVC video are in synch, } \\
T_{I_{V}}^{V}\left(j_{V}\right)-T_{I_{A}}^{A}\left(j_{A}\right)<-\eta_{-} & : \text {audio is ahead of SVC video, }
\end{array}
$$


where $\eta_{+}$and $\eta_{-}$are thresholds used for boundaries of the in-sync region. To apply this decision rule, it is evident that we need to inspect every RTCP packet for the computation of (1) through (5).

\section{Proposed Inter-media Synchronization Method}

Now we will derive the proposed scheme from the conventional method described by (1)-(5). In this study, we exploit the fact that after a connection has been setup, the codec type and the sampling rate are usually sustained during the connection.

By canceling out each term in the computation of $\sum_{k=2}^{j_{A}} \frac{\Delta M_{I_{A}}^{A}(k)}{R^{A}}$ in (1) and by using (2), we can simplify (1) into the following form

$$
\begin{aligned}
T_{I_{A}}^{A}\left(j_{A}\right) & =T_{I_{A}}^{A}(1)+\frac{M_{I_{A}}^{A}\left(j_{A}\right)-M_{I_{A}}^{A}(1)}{R^{A}} \\
& =T_{I_{A}}^{A}(0)+\frac{M_{I_{A}}^{A}\left(j_{A}\right)-M_{I_{A}}^{A}(0)}{R^{A}} .
\end{aligned}
$$

Assuming that $R^{A}$ is kept as a constant, we can obtain (7) from the NTP and RTP timestamps carried by the 0 -th and the $I_{A}$-th RTCP packet as follows

$$
R^{A}=\frac{M_{I_{A}}^{A}(0)-M_{0}^{A}(0)}{T_{I_{A}}^{A}(0)-T_{0}^{A}(0)} .
$$

Rearranging (7) for $T_{I_{A}}^{A}(0)$ and substituting it into (6) yields

$$
T_{I_{A}}^{A}\left(j_{A}\right)=T_{0}^{A}(0)+\frac{M_{I_{A}}^{A}\left(j_{A}\right)-M_{0}^{A}(0)}{R^{A}} .
$$

Similarly, we can apply this procedure to obtain the relation (9) for the RTP stream of SVC video session.

$$
T_{I_{V}}^{V}\left(j_{V}\right)=T_{0}^{V}(0)+\frac{M_{I_{V}}^{V}\left(j_{V}\right)-M_{0}^{V}(0)}{R^{V}} .
$$

By subtracting (9) from (8) and applying this result to (5), we can derive the following compact decision rule (10) after some arithmetic,

$$
\begin{aligned}
& d>\eta_{0}+R^{A} R^{V} \eta_{+} \quad: \text { SVC video is ahead of audio, } \\
& \eta_{0}+R^{A} R^{V} \eta_{+} \geq d \geq \eta_{0}-R^{A} R^{V} \eta_{-} \quad: \text { audio and SVC video are in sync, (10) } \\
& d>\eta_{0}-R^{A} R^{V} \eta_{-} \quad: \text { audio is ahead of SVC video, }
\end{aligned}
$$

where the variable $d$ and the threshold constant $\eta_{0}$ are defined by

$$
\begin{gathered}
d=R^{A} M_{I_{V}}^{V}\left(j_{V}\right)-R^{V} M_{I_{A}}^{A}\left(j_{A}\right), \\
\eta_{0}=R^{A} R^{V}\left(T_{0}^{V}(0)-T_{0}^{A}(0)\right)+R^{V} M_{0}^{A}(0)-R^{A} M_{0}^{V}(0) .
\end{gathered}
$$

Note that we only need to compute $d$ by (11) when examining the synchronization of each pair of RTP packet by (10). The reason is that $\eta_{0}+R^{A} R^{V} \eta_{+}$and $\eta_{0}-R^{A} R^{V} \eta_{-}$in (10) are needed to be computed just once after receiving the first RTCP packet. Since all of the $R^{V}, R^{A}, M_{I_{V}}^{V}\left(j_{V}\right)$, and $M_{I_{A}}^{A}\left(j_{A}\right)$ values in (11) are fixed-point numbers themselves, there is no need to utilize floating-point operations at all. Obviously, this is a 
clear advantage for embedded processors, which usually do not have floating point units. Moreover, (11) does not require any division operations like the case of (1)-(4). For ARM processors, avoiding division is a great advantage, since they do not have any hardware divider. In [1], they reported that truncation round-off errors may accumulate, since their method is computed by repeated divisions and summations. However, it is obvious that there are no possibilities of error accumulation in the computation of (11). Note that only two fixed-point multiplications and one subtraction are needed for the computation of $d$ in (11).

\section{Conclusions}

In this paper, we addressed the problem of synchronization for SVC video transport over IP networks. The synchronization issue includes layer synchronization among scalable layers of SVC video and inter-media synchronization between audio and SVC video. It has first been discussed non-interleaved mode of RTP packetization is suitable to provide layer synchronization among scalable layers of SVC video. Then, we proposed a computationally simple RTCP packet processing for inter-media synchronization. The advantageous aspects of the proposed method can be summarized in three ways. First, the decision rule is far simpler than the conventional method. Second, it does not require RTCP SR packet processing for synchronization except first RTCP packet. Finally, the proposed method does not suffer from the accumulation of round-off errors that are inherent in the conventional method.

Acknowledgements. This work was supported in part by the Gangwon-Alberta Research Collaboration Fund.

\section{References}

1. Bertoglio L., and Migliorati P.: Intermedia synchronization for video conference over IP. Signal Processing: Image Communication, Vol. 15, No. 1, (1999) 149-164.

2. Wenger S., Hannuksela M., Westerlund M., Singer D.: RTP payload format for H.264 video. RFC 3984, IETF, (Feb. 2005).

3. Wenger S.: RTP payload format for SVC video. IETF Internet Draft: draft-wenger-avt-rtpsvc-03.txt, (Oct. 2006).

4. Reichel J., Schwarz H., and Wien M.: Scalable video coding- Working Draft 3. JVT-P201, Poznan, Poland . (July 2005,)

5. Schulzrinne H., Casner S., Frederick R., and Jacobson V.: Real-time transport protocol. RFC 3550, IETF, (July 2003). 